\title{
Application of Mechanical Springs as Inductive Position Sensors
}

\author{
Hendrik Zur ${ }^{1}$, Fabian Wießner ${ }^{2}$ \\ ${ }^{1}$ Hochschule für Technik, Wirtschaft und Kultur Leipzig (HTWK), \\ Karl-Liebknecht-Str. 132, 04277 Leipzig, \\ hendrik.zur@eit.htwk-leipzig.de \\ ${ }^{2}$ Forschungs- und Transferzentrum Leipzig e.V. (FTZ), \\ Wächterstr. 13, 04107 Leipzig, Germany
}

\section{Summary:}

In many fields of technology, attempts are made to use affordable sensors for the monitoring of buildings or technical facilities. These sensors should have a high long-term stability. This paper illustrates how to use mechanical springs as sensors by measuring the inductance of the spring. It describes the concept of the sensor and the possibilities of dimensioning and computation.

Key words: mutual inductance, mechanical spring, cost reduction.

\section{Motivation}

Springs have been used for a long time in human history. They are characterized by good mechanical properties and their ability to store energy. But it is not just the mechanical properties that offer advantages, as it is also possible to measure the extension of a mechanical spring by using its electrical properties, especially its inductance. This effect can be used to design inductive position sensors.

\section{Sensor Concept}

The inductance of a spring varies in inverse proportion to its length. By measuring the inductance, the spring can be used as a sensor. The advantage of this kind of sensor is that no mechanical rebound elements are needed as the spring is able to get back to its original condition. Based on this, the sensor can replace elastic elements in mechanical structures. This will result in a reduction of costs thanks to the lower number of components used. Furthermore, the sturdy design of mechanical springs enables their use in industrial environments.

\section{Example of a Spring Sensor}

The concept is applied in an inductive position sensor (Fig. 1) developed by the FTZ and Scherdel Innotec. The sensor consists of a onepiece double spring. The inner and outer windings have the same electrical sense of direction. The advantages of this double spring are that the absolute change of its inductance is greater and that its endings are closer, which makes it easier to connect them with the electronic component of the sensor (Fig. 2).

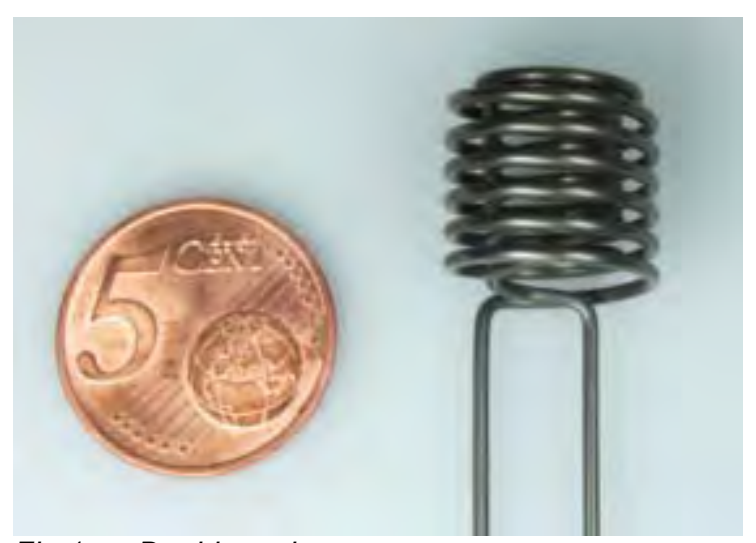

Fig.1. Double spring 


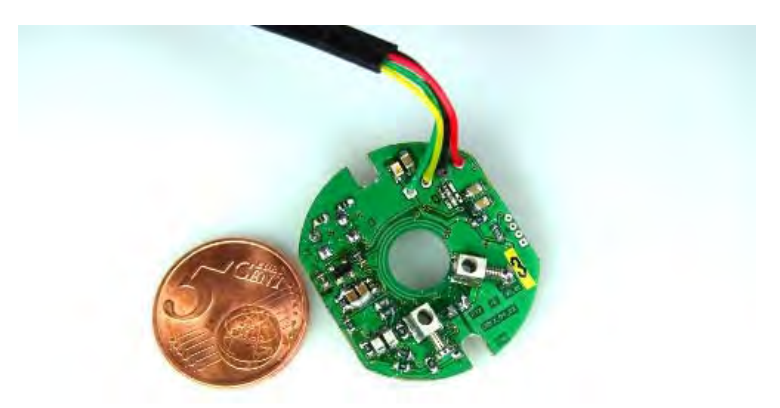

\section{Computation}

To determine the self-inductance of a mechanical spring, one may specify an electrical current $I$, compute the vector potential (eq. 1) along the inner contour $c_{2}$ (Fig. 3) of the spring path [1] and integrate it along this contour (eq. 2).

$$
\begin{aligned}
& \vec{A}(\vec{r})=\frac{\mu_{0} I}{4 \pi} \int_{c_{1}} \frac{\vec{d} r^{\prime}}{\left|\vec{r}-\vec{r}^{\prime}\right|} \\
& \Phi=\int_{c_{2}} \vec{A}(\vec{r}) \vec{d} r
\end{aligned}
$$

This leads to the value of magnetic flux. In order to obtain the value of external inductance, the magnetic flow has to be divided by the specified current (eq. 3).

$$
L_{e}=\frac{\Phi}{I}
$$

The inner inductance is approximated by

$$
L_{i}=\frac{\mu_{0} \mu_{r} l}{8 \pi}
$$

while $l$ is the length of the wire and $\mu_{r}$ is the relative permittivity of the spring material. The self-inductance $L$ is calculated by summarising the inner inductance $L_{i}$ and the external inductance $L_{e}$.

$$
L=L_{i}+L_{e}
$$

The following figure (Fig. 3) illustrates how the
Fig. 2. Electronic component of the sensor

external inductance of a single winding is calculated.

\section{Fig. 3. Calculation of a single winding}

For reasons to do with the calculation, the software uses models that consist of many separate segments. Because of this, the integrals (eq. 1 and eq. 2) are turned into sums. The number of segments depends on the number of windings as every winding is divided into a constant amount of parts.

This kind of calculation has, of course, its limitations. First, it requires that the spring be enclosed in a homogeneous, non-conducting medium. Second, the inductance results are incorrect when it comes to high frequency problems, because skin effect and proximity effect are not considered here. Furthermore, a non-ferromagnetic spring material is expected.

\section{Software}

The software tool (Fig. 4) was implemented with particular regard to the computation of the inductance of spiral springs for low frequency problems. It was developed using the programming language Java so it can be executed in various operating systems.

First, the user has to design a mechanical spring. After the geometrical parameters, such as material, diameter, height and number of windings, have been defined, the software generates a three-dimensional model. It is visualized as a wire, which means the conductor diameter is not visible.

The computation of the external inductance itself uses multiple threads. Based on this, the software tool takes up more of the processor's capacity but in turn delivers the calculation results much faster. 


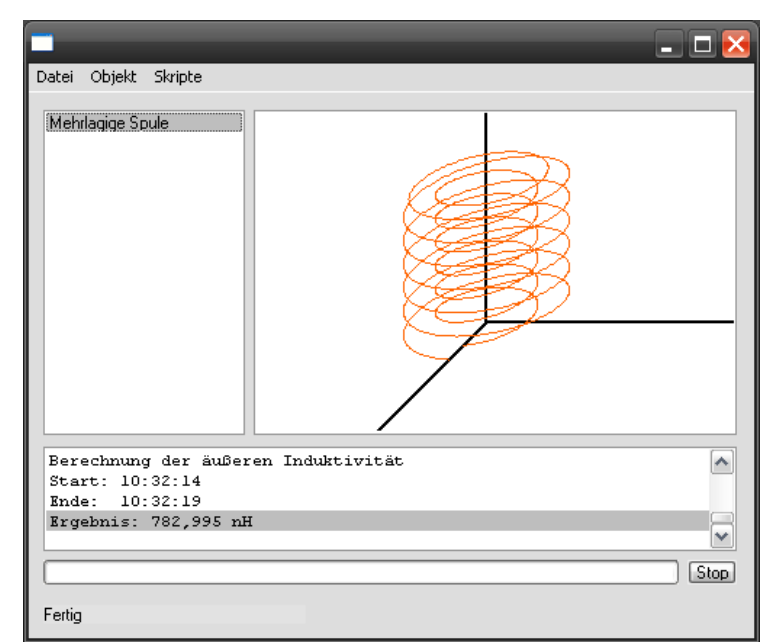

Fig. 4. Software tool (example)

In addition to the calculation results of the inner inductance and the external inductance, the software displays the resistance, the weight of the spring and the length of the wire. Furthermore, the spring rate of cylindrical springs can be calculated.

The advantages of the software tool are easy modelling, fast calculation results and the ability to compute mutual inductions.

\section{Simulation Example}

As an example, the self-inductance of a spring (Fig. 5) with the following parameters was calculated in the software tool and compared to the result of an FEM simulation:

$\begin{array}{ll}\text { Number of windings: } & 6.75 \\ \text { Height: } & 28 \mathrm{~mm} \\ \text { Diameter: } & 12 \mathrm{~mm} \\ \text { Conductor diameter: } & 1.2 \mathrm{~mm}\end{array}$

In both software applications, copper was the material of choice as it has a relative permittivity of about 1 . The surrounding medium was also assumed to have a relative permittivity of 1 . The calculated self-inductance values, including internal and external inductance, were as follows:

$\begin{array}{ll}\text { Result from the FEM: } & 196.78 \mathrm{nH} \\ \text { Result from the software tool: } & 195.23 \mathrm{nH}\end{array}$

In conclusion, a very high conformity was achieved, but the software tool calculation was much faster. It took just two seconds to calculate self-inductance.
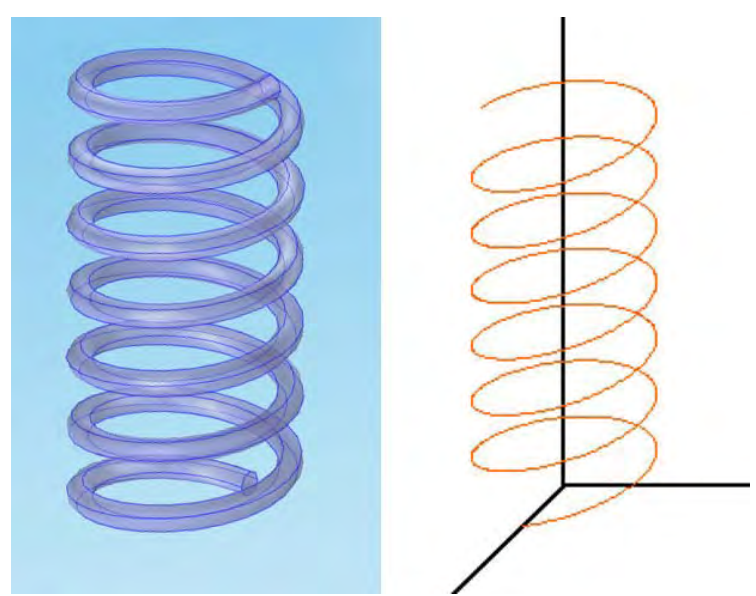

Fig. 5. right: FEM model, left: polygonal model

\section{Results}

As described in the example section above, the software tool delivers good results with very fast times. Currently, the calculation time increases proportionally to the second power of the number of segments. Because of this, the software still needs to be optimized for the computing of larger models.

For the future, there are plans to design inductive spring sensors using the software tool. Therefore, the spring rate has to be computed as well. The result will be a software tool that generates virtual spring models by combining their electrical and mechanical properties.

\section{References}

[1] Küpfmüller, Karl; Kohn, Gerhard: Theoretische Elektrotechnik ed. 14 Berlin: Springer, 1993

[2] Eichhorn, Karl Fr.: Induktivitäten windschiefer Leitersysteme (Archiv für Elektrotechnik Vol. 61) Berlin: Springer, 1979 